\title{
Condiciones ambientales a finales del Estadio Isotópico 6 (EI 6: > 130000 años) en el centro de México: caracterización de una sección de sedimentos laminados proveniente del Lago de Chalco
}

\author{
Diana Angélica Avendaño-Villeda ${ }^{1}$, Margarita Caballero ${ }^{2, *}$, Beatriz Ortega-Guerrero ${ }^{3}$, \\ Socorro Lozano-García ${ }^{3}$ y Erik Brown ${ }^{4}$
}

${ }^{1}$ Posgrado de Ciencias de la Tierra, Instituto de Geofísica, Universidad Nacional Autónoma de México, Ciudad Universitaria, C.P. 04510, Ciudad de México, México.

${ }^{2}$ Laboratorio de Paleolimnología, Instituto de Geofísica, Universidad Nacional Autónoma de México, Ciudad Universitaria, C.P. 04510, Ciudad de México, México.

${ }^{3}$ Instituto de Geología, Universidad Nacional Autónoma de México, Ciudad Universitaria, C.P. 04510, Ciudad de México, México.

${ }^{4}$ Large Lakes Observatory, University of Minnesota, Duluth, Minnesota 55812, EE.UU.

*maga@igeofisica.unam.mx.

\section{RESUMEN}

En los sistemas lacustres relativamente profundos y con un bajo contenido de oxígeno en la interfaz agua-sedimento es común la preservación de sedimentos anualmente laminados (varvas), los cuales indican una estacionalidad marcada en los procesos de depósito. El objetivo de este trabajo es caracterizar una sección laminada del registro sedimentario del Lago de Chalco, cuenca de México, que se presenta intercalada con sedimentos masivos, material volcaniclástico y 13 capas de lodo micrítico. Esta secuencia laminada se ubica entre los 122.5 y los $106 \mathrm{~m}$ de profundidad y se le ha asignado una edad mayor a $130 \mathrm{ka}$, periodo que coincide con el final del penúltimo máximo glacial, o Estadio Isotópico 6 (EI 6). La caracterización de los sedimentos se realizó mediante la observación al microscopio estereoscópico, compuesto y petrográfico de preparaciones frotis para identificar los componentes de las láminas claras, láminas oscuras y capas de lodo micrítico; algunas láminas claras, oscuras y capas de lodo micrítico fueron observadas con un microscopio electrónico de barrido. También se reportan datos de contenido de Ti y relaciones $\mathrm{Si} /$ Ti y Ca/Ti obtenidos mediante fluorescencia de rayos-X. Estos análisis permitieron identificar que las láminas claras tienen altos valores de Si/Ti y están formadas mayoritariamente por valvas de diatomeas (Stephanodiscus niagarae y S. oregonicus), por lo que se definen como una facies biogénica de lodo (ooze) de diatomeas. Las láminas oscuras tienen elevados valores de Ti y están formadas mayoritariamente por material clástico que incluye plagioclasas y anfíboles. Se propone un modelo de deposición de láminas anuales, en el cual la lámina clara corresponde con un florecimiento invernal de diatomeas mientras que la oscura corresponde con el depósito de material detrítico durante la estación de lluvias de verano, formando un par de láminas anuales biogénica-clástica o varva. La ecología de las especies dominantes indica que el depósito de la secuencia laminada corresponde con un lago relativamente profundo, eutrófico a hipertrófico de aguas dulces, templadas a frías. Esto es consistente con un escenario donde el depósito laminado correlaciona con el final del EI 6, durante el cual se interpretan condiciones más frías y húmedas que durante el EI 2 (último máximo glacial). La tasa de sedimentación calculada mediante el conteo de varvas arroja un promedio de $2.9 \mathrm{~mm} /$ año y permite estimar que el depósito de este tipo de sedimentos ocurrió a lo largo de un intervalo de $\sim 5000$ años. Las capas de lodo micrítico tienen altas relaciones de $\mathrm{Ca} / \mathrm{Ti}$ y $\mathrm{Si} / \mathrm{Ti}$ y están formadas principalmente por carbonatos autigénicos y por valvas de diatomeas, espículas de esponjas y valvas de ostrácodos. Estas capas representan facies químicas que se propone se depositaron durante momentos de mayor evaporación y mayor concentración química del agua, asociados con niveles lacustres más bajos y una alta productividad biológica. Dichas capas representan eventos climáticos recurrentes cuya periodicidad deberá ser estudiada más a fondo pero que considerando su espaciamiento en la secuencia y la tasa de sedimentación de $2.9 \mathrm{~mm} /$ año pudiera corresponder con un rango de 140 a 280 años.

Palabras clave: sedimentos laminados; varvas; Lago de Chalco; diatomeas; Estadio Isotópico 6 (EI 6), México.

\begin{abstract}
Annually laminated sediments (varves) frequently form in lakes with contrasting seasonal environments, and are better preserved in deep water bodies, with low oxygen content at the water/sediment interface. We characterized deposits in a laminated section within the sedimentary record from Lake Chalco, Basin of Mexico. The laminated sediments are intercalated with massive sediments, volcaniclastic material and 13 micritic mud layers. This laminated sequence corresponds to the depth interval 122.5 to $106 \mathrm{~m}$ and has an age $>130 \mathrm{ka}$, corresponding with the end of the penultimate glacial stage or Marine Isotopic Stage 6 (MIS 6).
\end{abstract}

Avendaño-Villeda, D.A. Caballero, M., Ortega-Guerrero, B., Lozano-García, S., Brown, E., 2018, Condiciones ambientales a finales del Estadio Isotópico 6 (EI 6: $>130000$ años) en el centro de México: caracterización de una sección de sedimentos laminados proveniente del Lago de Chalco: Revista Mexicana de Ciencias Geológicas, v. 35, núm. 2, p. 168-178. 
Dark and light lamina, as well as the micritic mud components, were identified by microscopic observation of sediment smear slides; some sediment in the dark laminae, light laminae and micritic mud layers was also analyzed using a scanning electron microscope. X-ray fluorescence was used to determine Ti content and Si/Ti and $\mathrm{Ca} / \mathrm{Ti}$ ratios. These analyses enabled us to determine that the light laminae have high $\mathrm{Si} / \mathrm{Ti}$ values, are formed mostly by diatom valves (Stephanodiscus niagarae and $S$. oregonicus), and represent a biogenic diatom ooze. Dark laminae have high Ti values and are formed mainly by clastic sediments including plagioclases and amphiboles. We propose an annual depositional model in which light laminae correspond to winter diatom blooms, whereas dark laminae were deposited by allochthonous inwash during the summer rainy season, thus forming a biogenic-clastic varve. Diatom ecology suggests the laminated sequence corresponds to a relatively deep, eutrophic to hypertrophic lake with cool to cold waters. This inference is consistent with the age of the deposits, which corresponds with the penultimate glacial maximum or MIS 6, for which we infer cooler and moistier conditions than for the last glacial maximum i.e. MIS 2. Sedimentation rates estimated by varve counts suggest that the laminated sequence was deposited during a 5000 yr interval. The micritic mud layers have high $\mathrm{Ca} / \mathrm{Ti}$ and Si/Ti ratios and are formed mainly by authigenic carbonates, sponge spicules, diatoms and ostracode valves. We propose that these chemical facies were deposited during periods of higher evaporation and water concentration and also lower lake level and high biological productivity. The recurrence of these events must be further studied, but based on the mean sedimentation rate of $2.9 \mathrm{~mm} / \mathrm{yr}$, calculated from varve counting we suggest that they have a 140-yr to 280-yr recurrence interval.

Key words: laminated sediments; varves; Lake Chalco; diatoms; Marine Isotope Stage 6 (MIS 6), Mexico.

\section{INTRODUCCIÓN}

Los registros sedimentarios en los lagos son fuentes de información que permiten conocer las fluctuaciones climáticas y ambientales a partir del estudio de sus componentes y de este modo proporcionan información sobre los factores y procesos que ocurren tanto fuera como dentro de ellos (Cohen, 2003). Dependiendo del origen de los sedimentos, éstos se clasifican en clásticos, biogénicos y químicos (Schnurrenberger et al., 2003). Los sedimentos clásticos están compuestos por partículas minerales y/o fragmentos de rocas que son arrastradas por el viento o por corrientes de agua superficiales hacia el cuerpo de agua. Los sedimentos biogénicos se forman a partir de estructuras orgánicas, calcáreas o silíceas, de algas, plantas o animales (e.g. polen, esporas, diatomeas, ostrácodos, etc.). Los sedimentos químicos son aquellos minerales que precipitan en la columna de agua o se forman en la interfaz agua-sedimento por procesos de diagénesis temprana. En un ambiente de depósito determinado, se acumulan diferentes proporciones de cada fracción originando paquetes sedimentarios con características físicas, biológicas y químicas particulares que son el resultado del ambiente y condiciones de formación; al conjunto de características intrínsecas del sedimento que permiten asociarlo con su ambiente de formación se le conoce como facies sedimentarias.

En los sedimentos lacustres es frecuente la presencia de laminaciones (capas de $<1 \mathrm{~cm}$ de espesor) que se depositan de forma recurrente, alternando dos o más tipos de facies sedimentarias que pueden ser predominantemente de origen clástico, biogénico o químico. A estas secuencias laminadas se les conocen como ritmitas, y su depósito puede estar asociado a factores y dinámicas climáticas y/o ambientales recurrentes en escalas multianuales, sin embargo, en los sistemas lacustres lo más común es que las láminas se depositen como respuesta a cambios estacionales de ciclicidad anual (Kemp, 1996; Ojala et al., 2012). Estas laminaciones con frecuencia consisten en la alternancia de una lámina oscura y otra clara, y al conjunto de láminas que representan un ciclo anual se les denomina varva (De Geer, 1912; Anderson y Dean, 1988; Zolitschka et al., 2015). Cada tipo de laminaciones es favorecido por condiciones ambientales específicas, por ejemplo, las laminaciones de origen clástico se forman por la entrada de material terrígeno, por acarreo de la escorrentía superficial por deshielos o lluvias intensas, y por lo tanto son más comunes en ambientes húmedos. En el caso de las biogénicas, se forman por acumulaciones masivas de restos de organismos asociados con aportes estacionales de nutrientes, siendo los más comunes los florecimientos algales favorecidos por una mezcla de la columna de agua. Las laminaciones de origen químico están formadas por la precipitación de minerales en la columna de agua (calcita, aragonita, yeso, etc.), siendo lo más frecuente la precipitación de calcita favorecida por una elevada evaporación en climas áridos y semiáridos y/o por el consumo de $\mathrm{CO}_{2}$ por una alta tasa de fotosíntesis durante florecimientos algales (Sáez et al., 2007; Zolitschka et al., 2015). Para la preservación de las laminaciones es necesaria la existencia de condiciones adecuadas, como la ausencia de bioturbación (alteración de los sedimentos por organismos bentónicos), la ausencia de re-suspensión de los sedimentos de fondo, o la ausencia de corrientes que interrumpan la sedimentación continua (Cohen, 2003; Brauer, 2004). Estas condiciones se presentan con mayor frecuencia en cuerpos de agua relativamente profundos $(>10 \mathrm{~m})$, en donde la acción del viento no llega al fondo, por lo que no se alteran los sedimentos por procesos mecánicos, y la columna de agua puede estratificar; bajo estas condiciones se desarrolla un hipolimnion que tiende a presentar condiciones anóxicas, que restringen la presencia de organismos bentónicos que perturben el sedimento. Otros factores que pueden favorecer la preservación de las laminaciones es la elevada concentración de materia orgánica (eutrofia a hipertrofia), que acelera el desarrollo de condiciones anóxicas dada la elevada demanda de oxígeno para degradar la materia orgánica, o condiciones de elevada salinidad, que por efecto de densidad hacen más estable la estratificación de un cuerpo de agua.

La preservación de secuencias laminadas en ambientes lacustres permite realizar estudios de alta resolución, en particular las varvas permiten el desarrollo de modelos geocronológicos en los que se puede llegar a tener una resolución estacional de las fluctuaciones climáticas (Brauer, 2004; Ojala et al., 2012). Este tipo de estudios han sido reportados con mayor frecuencia para lagos europeos, pero los sedimentos anualmente laminados (o varvados) tienen una distribución global. En México se han reportado sólo algunos lagos con sedimentos anualmente laminados que incluyen a Juanacatlán en los altos de Jalisco (Metcalfe et al., 2010), donde las varvas son de origen clástico-biogénico, y a la Hoya la Alberca y Hoya Rincón de Parangueo en Guanajuato (Kienel et al., 2009), Aljojuca en Puebla (Bhattacharya y Byrne, 2016) y Minucúa en Oaxaca (Goman et al., 2017), donde las varvas son predominantemente de origen clástico-químico.

Dentro de este contexto, en el que los registros laminados en el centro de México son relativamente escasos, destaca la presencia de sedimentos laminados en la secuencia lacustre del antiguo Lago de Chalco, en la cuenca de México. Estos sedimentos se localizan por lo menos desde los $122.5 \mathrm{~m}$ y hasta los $106 \mathrm{~m}$ en el sondeo CHA08, y se les ha asignado una edad mayor a 130000 años (Ortega-Guerrero et al., 2017). En este trabajo se presenta la caracterización de los componentes que conforman a las láminas claras y oscuras dentro de esta secuencia, y se establece un modelo paleoambiental para explicar su formación bajo la hipótesis de que representan varvas. 


\section{SITIO DE ESTUDIO}

En la cuenca de México, el emplazamiento del campo volcánico monogenético de la Sierra Chichinautzin hace 1.2 Ma (e.g., Arce et al., 2013) obstruyó el antiguo drenaje al sur, lo que le dio el carácter de endorreica. Esto permitió la formación de grandes cuerpos lacustres: al norte Zumpango y Xaltocan, al centro Texcoco, al sur Xochimilco y al sureste Chalco (Rodríguez-Castillo y González-Morán, 1989; DíazRodríguez, 2006) (Figura 1). La Subcuenca de Chalco abarca alrededor de $240 \mathrm{~km}^{2}$ de planicie lacustre, delimitada al norte por la Sierra de Santa Catarina, al este por la Sierra Nevada (cadena volcánica que incluye al Iztaccíhuatl y Popocatépetl), al sur por el campo volcánico monogenético de la Sierra Chichinautzin y al oeste por la divisoria entre el Volcán Teuhtli y la Sierra Santa Catarina, que la separa de la Subcuenca de Xochimilco (Figura 1).

El clima actual en la cuenca de México se encuentra dominado por la migración al norte de la Zona de Convergencia Intertropical durante el verano, cuando hay una precipitación máxima asociada con los Vientos Alisios que transportan humedad proveniente del Golfo de México y el Caribe. El invierno, por otro lado, es relativamente seco y se caracteriza por un descenso de la temperatura, con la presencia de fuertes vientos y lluvias asociados con la entrada de frentes fríos (Mosiño-Alemán y García, 1974). La Subcuenca de Chalco, con una altitud de $2240 \mathrm{~m}$ s.n.m., presenta una temperatura media anual de $16.8^{\circ} \mathrm{C}$ y una precipitación total anual de $540 \mathrm{~mm}$.

\section{CONTEXTO PALEOAMBIENTAL}

Durante el Cuaternario el clima del planeta se caracterizó por una serie de fluctuaciones climáticas cíclicas en las que alternaron condiciones glaciales (frías) e interglaciales (cálidas); estas alternancias climáticas en la literatura son identificadas como estadios isotópicos (EI) debido a que se registran claramente en la señal de isótopos de oxígeno en carbonatos biogénicos acumulados en sedimentos marinos de diferentes cuencas oceánicas (Lisiecki y Raymo, 2005). Dentro de estas glaciaciones cíclicas destacan los dos últimos máximos glaciales que se identifican como EI 6, fechado en hace $~ 140000$ años y que representa el penúltimo máximo glacial y el EI 2, fechado en hace $\sim 20000$ años, que representa el último máximo glacial. Datos paleoambientales del SW de EEUU indican que en esta región, en donde actualmente dominan climas semiáridos, existieron climas relativamente húmedos durante los dos últimos máximos glaciales (EI 6 y EI 2), asociados con niveles lacustres altos, indicando condiciones de baja evaporación y una mayor precipitación de invierno en esta zona (Brook et al., 2006; Reheis et al., 2014). Los datos para Europa y la región circum-Mediterránea indican que el EI 6 fue un intervalo durante el cual la extensión de los casquetes de hielo en las altas latitudes fue mayor que durante el EI 2, y del que se tienen evidencias de climas tropicales relativamente húmedos comparados con el EI 2, durante el cual los trópicos fueron en general más secos (Ayalon et al., 2002; Roucoux et al., 2011). Sin embargo, existen pocos registros paleoambientales directamente de los trópicos que documenten las características climáticas del EI 6.

En el centro de México hay extensos registros de glaciares antiguos en las elevaciones más altas del país que dan evidencia de un descenso importante en la temperatura durante las dos últimas glaciaciones del Cuaternario (Vázquez-Selem y Heine, 2011). El registro glacial más completo corresponde al Iztaccíhuatl, del que destaca el avance glacial denominado Hueyatlalco-1, ocurrido durante el EI 2 y, durante el cual la altitud de la línea de equilibrio de los glaciares (ALEG) descendió de su posición moderna de 4970 m s.n.m., hasta los 3940 m s.n.m., y el término de los glaciares (que hoy se ubica a los $4860 \mathrm{~m} \mathrm{s.n.m.)}$ alcanzó los 3390 m s.n.m. No obstante, el avance glacial que tuvo la mayor extensión fue el Nexcoalango, correspondiente al EI 6, durante el cual el nivel de la ALEG fue menor a los 3900 m s.n.m. y el término de los glaciares llegó a los 3000 m s.n.m. (Vázquez-Selem, 2011). Este avance glacial no se tiene tan bien estudiado como el Hueyatlaco-1 debido a la erosión de sus estructuras sedimentarias, sin embargo, Vázquez-Selem y Heine (2011) estiman que la extensión de los glaciares durante el EI 6 era de más de $200 \mathrm{~km}^{2}$, esto es significativamente más extenso que en la actualidad $\left(1.3 \mathrm{~km}^{2}\right)$ y casi el doble que durante el EI 2 , cuando se estima que el área cubierta por glaciares era de $131 \mathrm{~km}^{2}$. Esta amplia extensión de los glaciares durante el EI 6 sugiere que en el

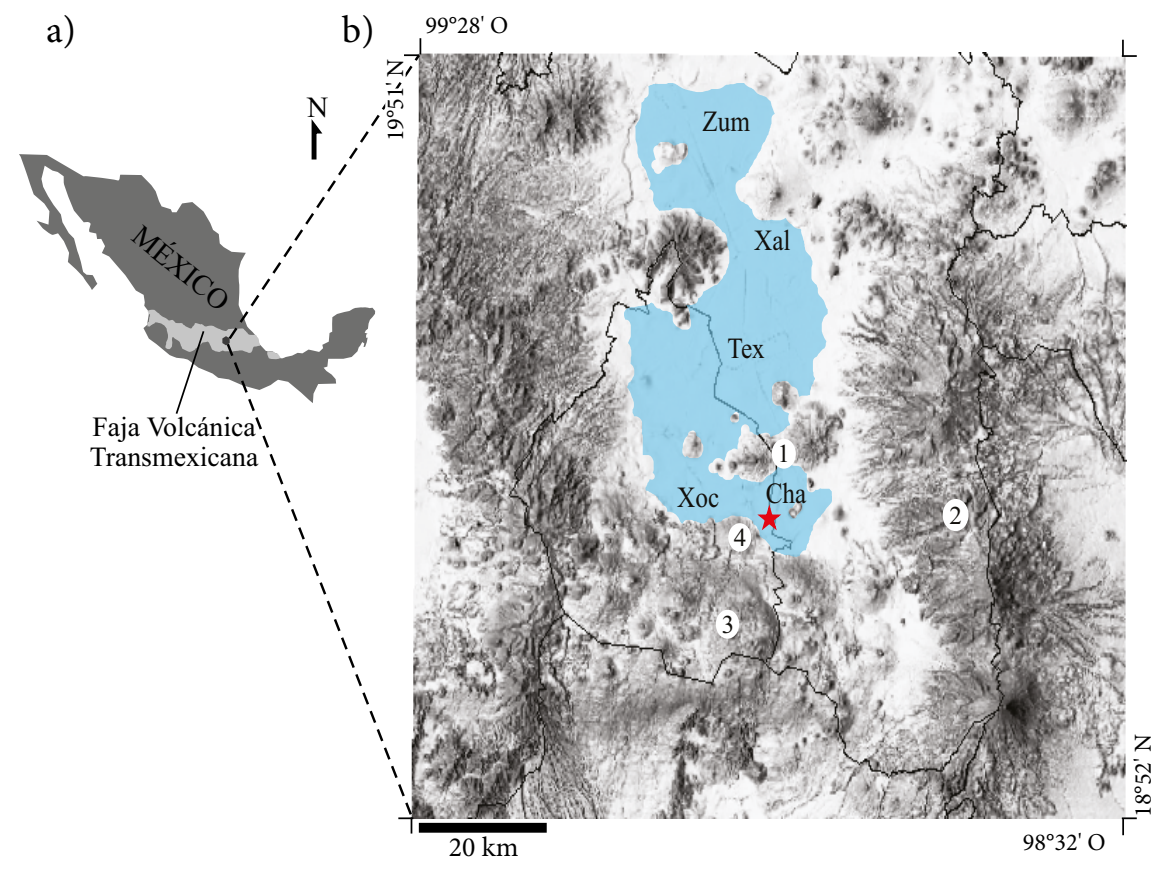

Figura 1. Localización del Lago de Chalco, al sureste de la cuenca de México, centro de México. a) ubicación de la cuenca de México dentro de la Faja Volcánica Transmexicana. b) cuenca de México con la extensión de los antiguos lagos: Zumpango (Zum), Xaltocan (Xal), Texcoco (Tex), Xochimilco (Xoc) y Chalco (Cha). Los números identifican los complejos volcánicos: Sierra Santa Catarina (1), Sierra Nevada (2), Sierra Chichinautzin (3) y Volcán Teuhtli (4). La estrella marca el sitio de perforación de la secuencias CHA08. 
centro de México el EI 6 fue probablemente más frío y húmedo que el EI 2, sin embargo, los registros paleoambientales para el EI 6 en esta región son sumamente limitados.

\section{MATERIALES Y MÉTODOS}

\section{Recuperación de la secuencia}

En 2008 se recuperaron cuatro secuencias sedimentarias paralelas cerca del depocentro de la Subcuenca de Chalco $\left(19^{\circ} 15^{\prime} 13^{\prime \prime N}\right.$, $98^{\circ} 58^{\prime} 39^{\prime \prime} \mathrm{O}$ ) (Figura 1b). En el presente trabajo se estudian los sedimentos de una de ellas, la secuencia CHA08-VI, que abarcó una profundidad entre los 122.5 a $71 \mathrm{~m}$. Todos los pozos fueron perforados y recuperados con un barril tipo Shelby, con camisa de PVC de 4" de diámetro interno, en secciones de $1 \mathrm{~m}$ de longitud, y posteriormente fueron transportados al National Lacustrine Core Facility (LacCore), de la Universidad de Minnesota, EE.UU., donde se abrieron y fotografiaron con una cámara digital de alta resolución DMT CoreScan. En conjunto los núcleos recuperados (CHA08) abarcan una secuencia de $122.5 \mathrm{~m}$ de profundidad máxima, cuya estratigrafía, sucesión de facies sedimentarias y modelo de edad han sido descritos previamente (Herrera-Hernández, 2011; Torres-Rodríguez et al., 2015; AvendañoVilleda, 2017; Ortega-Guerrero et al., 2017). Estos autores identificaron que en la parte basal de la secuencia existen sedimentos laminados entre los 122.5 y $106.0 \mathrm{~m}$ de profundidad (Figura $2 \mathrm{a}$ ) que son el objeto de estudio de este trabajo.

\section{Cronología}

El modelo de edad establecido para la secuencia de Chalco se basa en 15 fechamientos por radiocarbono en los $35.2 \mathrm{~m}$ superiores y un fechamiento por ${ }^{230} \mathrm{Th} /{ }^{234} \mathrm{U}$ a los $63.5 \mathrm{~m}$ que arroja una edad de 76700 años (Torres-Rodríguez et al., 2015; Ortega-Guerrero et al., 2017). Estos fechamientos permiten calcular tasas de sedimentación en Chalco que varían entre 0.2 y $12.5 \mathrm{~mm} / \mathrm{año}$, con una tasa promedio

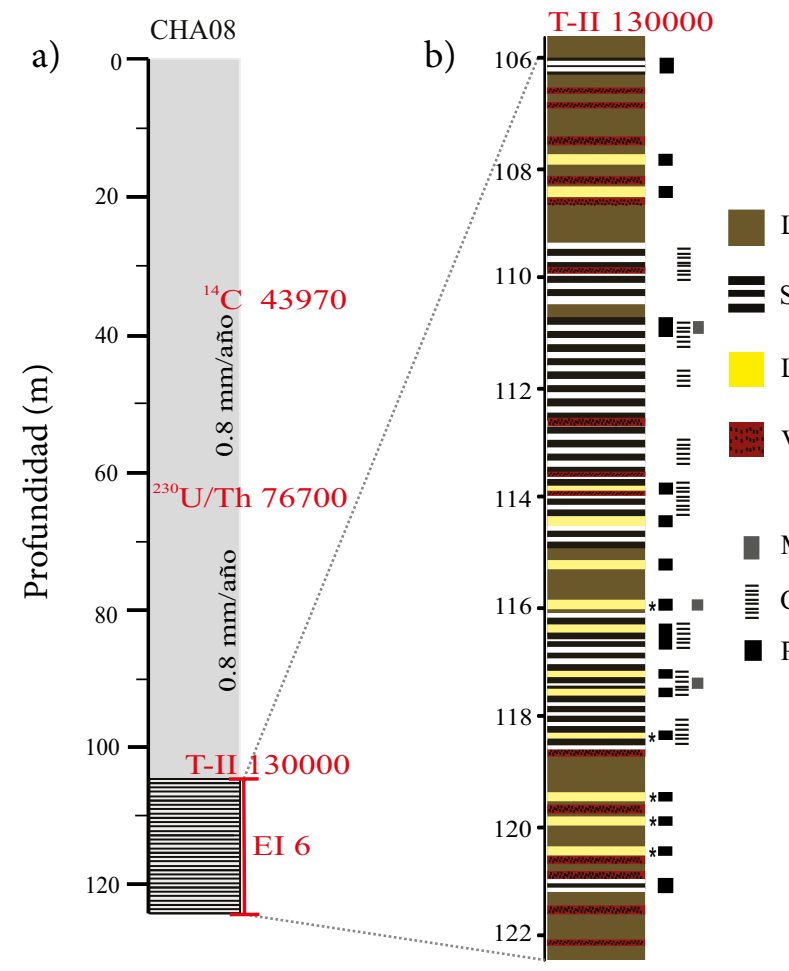

de $0.8 \mathrm{~mm} /$ año para los sedimentos que se ubican entre los 63.5 y los $35.2 \mathrm{~m}$. Extrapolando esta tasa de sedimentación y basándose en cambios abruptos en la asociación de facies, en los conjuntos de diatomeas y en los valores de la relación Ca/Ti, Avendaño-Villeda (2017) y OrtegaGuerrero et al. (2017) proponen que los $106 \mathrm{~m}$ de profundidad marcan la transición del EI 6 al EI 5, transición denominada Terminación II (T-II) y que ha sido fechada globalmente en 130000 años (Lisiecki y Raymo, 2005). Esta inferencia permite establecer que el paquete laminado corresponde al final del periodo glacial EI 6 y que tiene una edad mayor a 130000 años (Figura 2a).

\section{Fluorescencia de rayos - $\mathrm{X}$}

La composición elemental de la secuencia laminada fue determinada cada $1 \mathrm{~cm}$ mediante fluorescencia de rayos-X (FRX) utilizando el equipo ITRAX-XRF-Core Scanner (Cox Analytical Instruments) del Large Lakes Observatory de la Universidad de Minnesota-Duluth. En este trabajo se reportan los valores correspondientes al Ti y las relaciones $\mathrm{Si} / \mathrm{Ti}$ y $\mathrm{Ca} / \mathrm{Ti}$.

\section{Preparaciones frotis y observaciones al microscopio electrónico de barrido}

Para caracterizar los sedimentos que componen las láminas de la sección bajo estudio se realizaron 34 preparaciones frotis en secuencias de dos, cuatro u ocho laminaciones consecutivas claraoscura, este muestreo se distribuyó en cuatro diferentes intervalos de la sección laminada. También se realizaron 13 preparaciones frotis correspondientes a los horizontes de lodo amarillo pálido que están presentes entre los 122.5 y $106.0 \mathrm{~m}$ (Figura 2b). Los frotis se prepararon dispersando la muestra sobre un portaobjetos y montando con resina óptica Nordland (NOA61), la cual polimeriza al ser expuesta a luz UV. Las preparaciones frotis fueron analizadas bajo un microscopio estereoscópico (OLYMPUS SZX12) observando cinco campos visuales en 90x y 144x, y en un microscopio compuesto (OLYMPUS BX50) observando 10 campos visuales en 200x y 1000x. Para cada campo se estimó, de forma cualitativa, un porcentaje del área visual que ocupaban los fragmentos observados, dividiéndolos en dos categorías: 1) valvas o fragmentos de valvas de diatomeas, las cuales fueron identificadas a nivel de especie y 2 ) otros componentes. Además, se
Figura 2. Estratigrafía de la secuencia CHA08 del Lago de Chalco, cuenca de México. a) Ubicación de la sección laminada dentro de la estratigrafía general de la secuencia CHA08. b) Estratigrafía detallada de la secuencia laminada indicando los horizontes en los que se realizaron preparaciones frotis, conteos de laminaciones y muestreos para observaciones en el microscopio electrónico. El * identifica las capas de lodo amarillo pálido en las que el conjunto de diatomeas está dominado por Anomoeoneis costata y Campylodiscus clypeus. 
seleccionaron seis preparaciones correspondientes a láminas claras, cinco correspondientes a láminas oscuras y nueve a horizontes amarillo pálido para observarse en un microscopio petrográfico (400x y 600x MOTIC BA310 POL), con luz polarizada paralela y luz polarizada cruzada, con el objetivo de identificar los principales minerales presentes en cada tipo de sedimento.

Finalmente, se muestrearon dos secciones laminadas y un horizonte de lodo amarillo pálido (Figura 2b), utilizando cajas tipo Kubiëna de $3 \times 0.5 \times 1 \mathrm{~cm}$ en las cuales se recuperó el sedimento que posteriormente fue cubierto con oro para su observación a mayor detalle usando un microscopio electrónico de barrido (JEOL JCM-6000PLUS, bajo y alto vacío, 10 y $15 \mathrm{kV}$ ).

\section{Conteos de laminaciones}

Se realizaron ocho conteos de laminaciones a lo largo de la secuencia (Figura 2b) empleando las fotografías obtenidas de las secciones perforadas. Para realizar el conteo se utilizó el software Image J (Schneider et al., 2012), calibrándose las fotografías $(100 \mathrm{pxl} / \mathrm{cm})$ y se obtuvieron las bases de datos y las gráficas de escala de grises para cada perfil analizado. Esta escala de grises tiene como valores extremos 255 para blanco y 0 para negro. Para contar los pares de láminas claro-oscuro se determinaron los puntos de inflexión en cada curva, calculando su pendiente y aplicando un filtro móvil de 10 puntos (Boës y Fagel, 2008). Por último, para conocer el número de pares de láminas por intervalo, se contó de forma manual, por duplicado, cada pico de la curva de la pendiente. Con base en estos conteos, y considerando que cada par de laminaciones (clara y oscura) representan un año, es decir, que son varvas, se calculó una tasa de sedimentación para cada intervalo contado y se obtuvo un promedio. La sección laminada se encuentra interrumpida por depósitos volcaniclásticos y horizontes de lodo amarillo pálido, pero para el cálculo de las tasas de sedimentación los espesores de ambas facies se omitieron, ya que estos depósitos no forman parte de la secuencia varvada y no es posible estimar sus tiempos de depósito.

\section{RESULTADOS}

\section{Caracterización de la secuencia laminada}

La columna estratigráfica de Chalco contiene diferentes facies sedimentarias que alternan a lo largo de la secuencia, sin embargo, sólo aparece un intervalo laminado entre los 122.5 y $106 \mathrm{~m}$ de profundidad (Figura 2a) (Ortega-Guerrero et al., 2017). El intervalo que contiene las laminaciones presenta también capas decimétricas de facies de limo gris masivo rico en carbonatos, 13 capas de 2 a $5 \mathrm{~cm}$ de espesor de lodo amarillo pálido y 18 horizontes de depósitos volcaniclásticos félsicos y máficos de $0.5 \mathrm{a} 12 \mathrm{~cm}$ de espesor (Figura 2b). Sin embargo, lo que domina en este intervalo es una facies laminada que está constituida por conjuntos rítmicos de láminas claras y oscuras cuyo paquete principal se ubica entre los 119.0 y $109.5 \mathrm{~m}$, con paquetes menos potentes hacia la base (121.3-121.0 m) y cima (106.1-106.0 m) de la sección reportada (Figura 2b). Las laminaciones tienen diferente grado de preservación y en algunas secciones están claramente definidas mientras que en otras son más difusas o discontinuas. En las secciones que están mejor definidas (Figura 3a), las láminas presentan estratificación paralela planar con contactos abruptos y espesores variables que se mantienen entre 0.5 a $5 \mathrm{~mm}$, con secciones en las que dominan (son más gruesas) las láminas claras y otras en las que dominan las láminas oscuras. Sin embargo, hay diversas secciones en las que las laminaciones presentan contactos más difusos y su estratificación puede ser paralela, ondulada o discontinua. Es frecuente que lo que a simple vista parece ser una laminación más gruesa, al ser observada bajo mayor aumento se distingue como una sucesión de laminaciones de espesores milimétricos o sub-milimétricos.

Las láminas claras (Figura 3a y 3b) son de color pardo grisáceo, pero varían ampliamente en su tonalidad. Están compuestas en promedio (Tabla S1 del suplemento electrónico) por un $\sim 70 \%$ de valvas o fragmentos de valvas de diatomeas entre las que dominan Stephanodiscus niagarae Ehrenberg 1845 y S. oregonicus (Ehrenberg) Håkansson 1986 y en menor proporción Fragilaria capucina Desmazières 1830, Epithemia

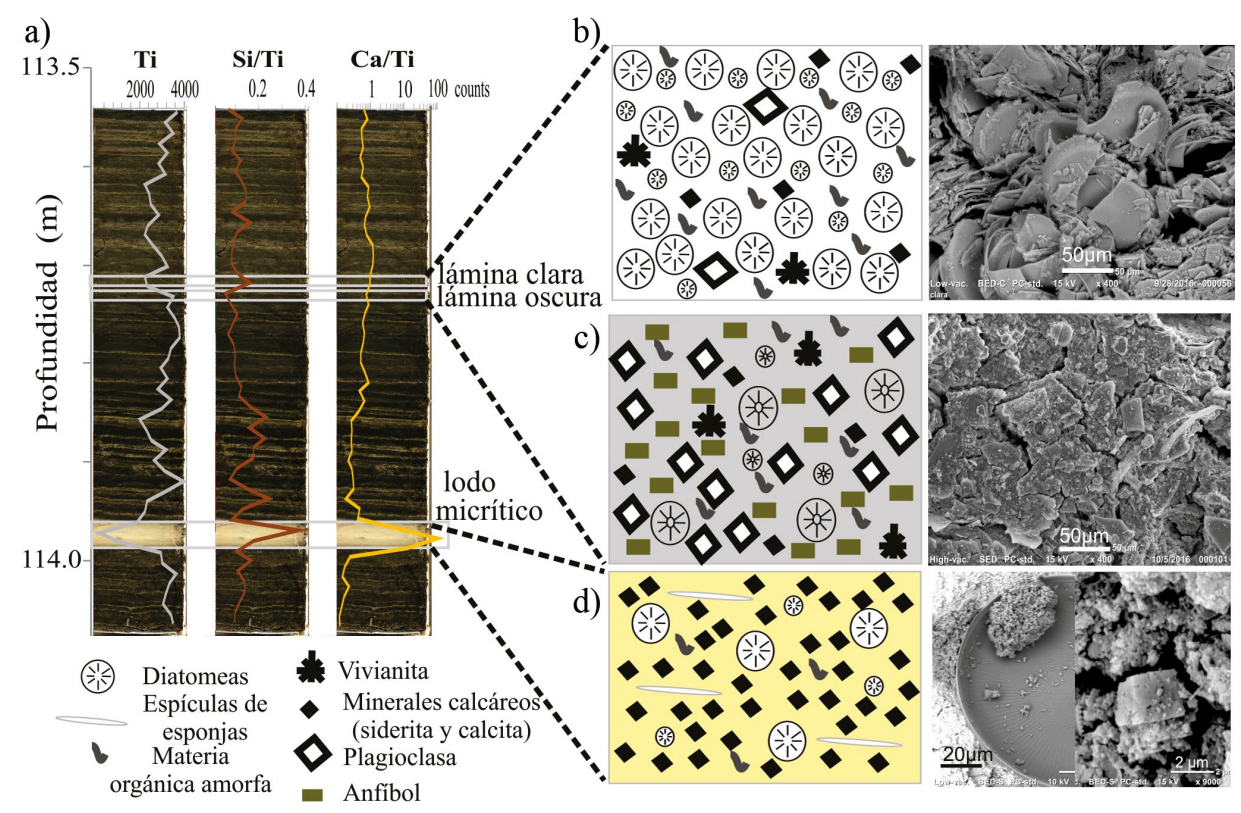

Figura 3. Fotografías, datos de fluorescencia de rayos-X, esquemas y micrografías al microscopio electrónico de barrido (MEB) que ilustran las características y composición del paquete de sedimentos laminados en la secuencia sedimentaria de Chalco. a) Aspecto general de una sección laminada con los valores de Ti, Si/ $\mathrm{Ti} \mathrm{y} \mathrm{Ca/Ti,} \mathrm{destacando} \mathrm{un} \mathrm{ejemplo} \mathrm{característico} \mathrm{de} \mathrm{una} \mathrm{lámina} \mathrm{clara,} \mathrm{una} \mathrm{lámina} \mathrm{oscura} \mathrm{y} \mathrm{una} \mathrm{capa} \mathrm{de} \mathrm{lodo} \mathrm{micrítico} \mathrm{amarillo} \mathrm{pálido} \mathrm{(nótese} \mathrm{la} \mathrm{escala} \mathrm{logarítmica}$ en los datos $\mathrm{Ca} / \mathrm{Ti}$ ). b) Esquema y microfotografía al MEB de una lámina clara. c) Esquema y microfotografía al MEB de una lámina oscura. d) Esquema y microfotografía al MEB de una capa de lodo micrítico. 
adnata (Kützing) Brébisson 1838 y Surirella peisonis Pantocsek 1902 (Figura $4 \mathrm{a}-4 \mathrm{e}$ ). Las láminas claras tienen en promedio un $\sim 30 \%$ de otros materiales que incluyen principalmente materia orgánica amorfa, minerales autigénicos como la siderita, vivianita y calcita y en menor proporción minerales clásticos como la plagioclasa.

Las láminas obscuras (Figura 3a y 3c) son de color gris oliva y están compuestas, en promedio (Tabla S2 del suplemento electrónico) por un $\sim 35 \%$ de valvas y fragmentos de valvas de diatomeas y $\sim 65 \%$ de otros materiales, entre los que destacan materia orgánica amorfa, minerales clásticos como anfíboles y plagioclasas (Figura 4f - 4i) y algunos minerales autigénicos como la calcita y vivianita (Figura $4 \mathrm{j}-4 \mathrm{~m}$ ). Cabe destacar que las especies de diatomeas presentes en las láminas oscuras son las mismas que en las láminas claras, esto es, están dominadas por S. niagarae y S. oregonicus.

Las capas de lodo amarillo pálido (Figura 3 a y $3 d$ ) están formadas (Tabla S3 del suplemento electrónico) en promedio, por un $\sim 25 \%$ de valvas de diatomeas y $75 \%$ de otros materiales entre los que dominan minerales autigénicos como calcita, siderita y ocasionalmente dolomita y también se identifica la presencia de espículas de esponjas. Las especies de diatomeas presentes en estas capas son variables, en la mayoría de las capas domina el mismo conjunto que en las laminaciones clarooscuro, formado por S. niagarae y S. oregonicus. Sin embargo, en cinco de las capas está presente un conjunto de diatomeas distinto, formado por Anomoeoneis costata (Kützing) Hustedt 1959 y Campylodiscus clypeus (Ehrenberg) Kützing 1844, además están presentes abundantes restos de valvas de ostrácodos. Las capas en las que se identifica este otro conjunto de diatomeas, en general, están intercaladas con horizontes de sedimento gris masivo y no con el sedimento laminado (Figura 2b).

\section{Fluorescencia de rayos- $\mathrm{X}$}

El análisis de FRX realizado tuvo una separación de $1 \mathrm{~cm}$ entre los puntos analizados, sin embargo, las laminaciones claro-oscuro son en general más delgadas, con espesores que varían entre 0.5 y $5 \mathrm{~mm}$, por lo que no es posible comparar los valores en laminaciones adyacentes. Sin embargo, si es posible correlacionar los valores obtenidos con la tonalidad de la lámina observada al cotejar las gráficas con las fotografías de la secuencia laminada correspondiente y así identificar tendencias en los valores de las capas claras y oscuras. De esta manera se puede determinar que las capas amarillo pálido y las láminas claras presentan valores relativamente bajos de Ti mientras que las láminas oscuras presentan valores elevados de este elemento (Figura 3a). El Ti es un elemento estable y tiene como único origen posible el material detrítico, lo que es consistente con la abundancia de minerales clásticos en estas láminas (Figura 3b).
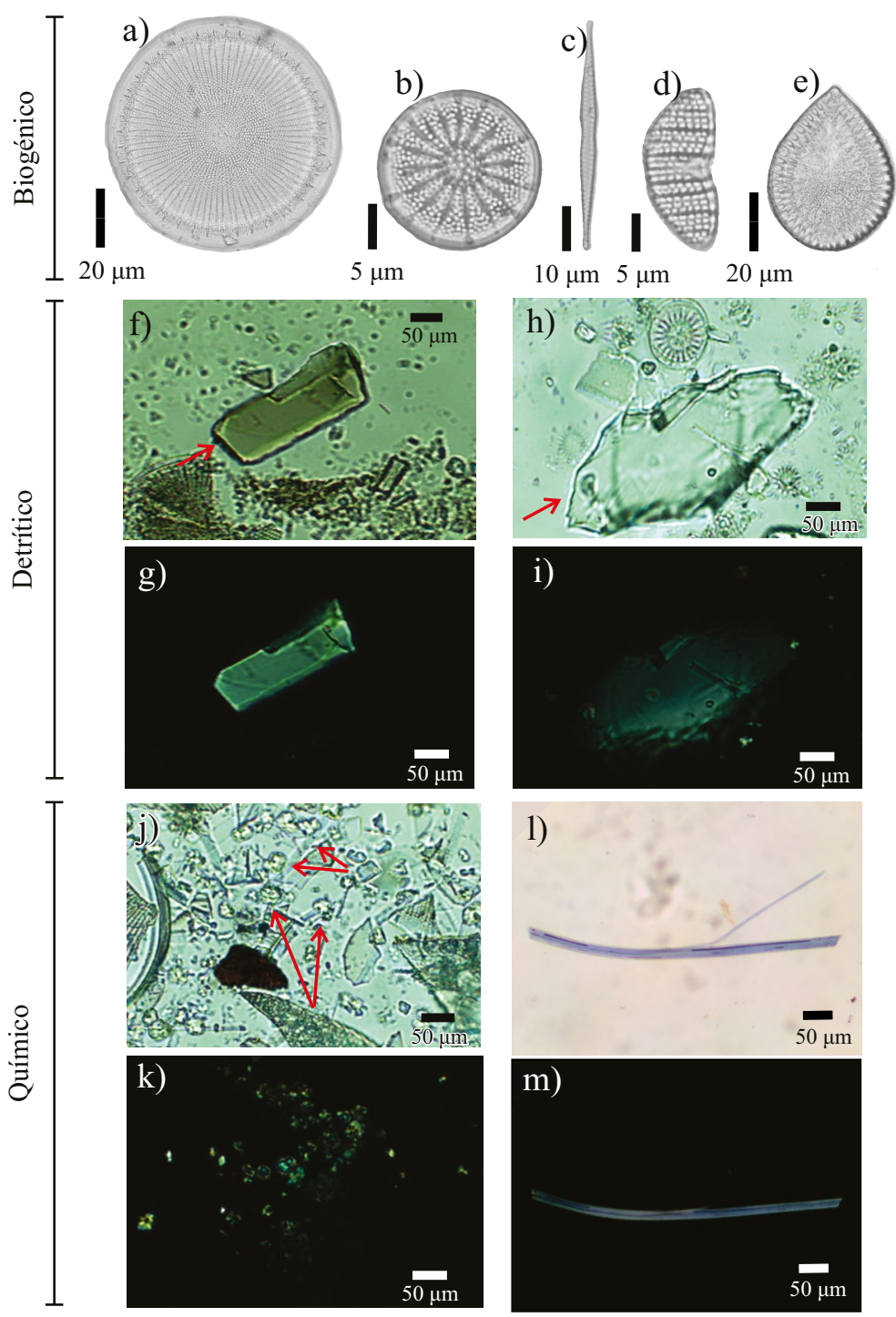

Figura 4. Componentes biogénicos (a-e), detríticos (f-i) y autigénicos (j-m) de los sedimentos laminados de Chalco, cuenca de México. Las imágenes (g), (i), (k) y (m) fueron obtenidas en el microscopio petrográfico con nicoles cruzados. a) Stephanodiscus niagarae, b) S. oregonicus, c) Fragilaria capucina, d) Epithemia adnata, e) Surirella paisonis, f-g) anfíbol, h-i) plagioclasa, j-k) calcita, l-m) vivianita. 
La relación de Si/Ti muestra una tendencia inversa a la del $\mathrm{Ti}$, con valores elevados en las láminas claras y valores relativamente bajos en las láminas oscuras (Figura 3a). Las bandas amarillo pálido, en general, también tienen valores altos de esta relación. La relación $\mathrm{Si} / \mathrm{Ti}$ es proporcional a la abundancia de sílice biogénico (Kylander et al., 2011) siendo las diatomeas y las esponjas de agua dulce los principales organismos silíceos en los lagos. El patrón observado, con valores $\mathrm{Si} / \mathrm{Ti}$ altos en la lámina clara, es consistente con las observaciones al microscopio tanto óptico como electrónico, donde se identificó que estas láminas están formadas mayoritariamente por valvas de diatomeas (Figura 3c). Los valores altos de Si/Ti en las capas amarillo pálido corresponden también con la presencia de abundantes fragmentos de valvas de diatomeas y espículas de esponjas (Figura 3d).

En cuanto a la relación $\mathrm{Ca} / \mathrm{Ti}$, estos valores son en general más bajos en las láminas oscuras, con valores más altos en las láminas claras; sin embargo, esta relación presenta valores particularmente elevados en las capas amarillo pálido (Figura 3a). La relación $\mathrm{Ca} / \mathrm{Ti}$ es proporcional a la presencia de carbonatos autigénicos, lo cual corresponde con la abundancia de minerales como la calcita y la presencia de abundantes valvas de ostrácodos en las capas amarillo pálido (Figura 3d).

\section{Conteos de laminaciones}

En las gráficas de escala de grises las láminas claras presentan valores que en general varían entre 70 a 150, mientras que las láminas oscuras tienen valores menores a 70. Las capas amarillo claro alcanzan valores de hasta 250, lo que las diferencia claramente de las láminas claras. En la Figura 5 se presenta un ejemplo de los conteos de laminaciones realizados en cada una de las ocho secciones analizadas y, en la Tabla 1 se presentan los resultados obtenidos en los ocho conteos, indicando las tasas de sedimentación para cada sección, considerando que representan varvas, y su promedio $(2.9 \mathrm{~mm} / \mathrm{año})$. Cabe mencionar que el amplio rango de valores en la tonalidad de las laminaciones claras representó una dificultad al momento de realizar el conteo ya que el contraste entre la lámina clara y oscura con frecuencia era bajo y, por lo tanto, los puntos de inflexión en las gráficas tanto de tonos de grises como las de sus pendientes eran pequeños. También, el hecho de que en secciones del núcleo dominaran los tonos claros u obscuros dificultó la identificación de algunas laminaciones dentro del contexto de una sección más o menos clara del núcleo. Es por ello que consideramos que las tasas de sedimentación estimadas en esta sección representan valores máximos, y que es muy probable que los valores reales sean menores.

\section{DISCUSIÓN}

El conjunto de diatomeas preservado en la secuencia laminada permite realizar una reconstrucción paleoambiental para la Subcuenca de Chalco hacia finales del EI 6. Las especies dominantes en el sedimento laminado, Stephanodiscus niagarae y S. oregonicus, son especies planctónicas de talla grande, que en general son favorecidas por una alta turbulencia en la columna de agua que permita su flotabilidad y que, por su baja relación superficie/volumen, requieren de altos niveles de nutrientes (Litchman et al., 2009; Bao et al., 2015). Estas especies habitan actualmente en lagos de EEUU y Canadá y pueden caracterizarse como especies planctónicas, de aguas dulces, templadas a frías, de profundidad intermedia $(\leq 15 \mathrm{~m})$, que toleran elevadas concentraciones de nutrientes (Theriot y Stoermer, 1981; Brugam, 1983; Theriot y Stoermer, 1984; Fritz et al., 1993; Bradbury, 1997; Julius et al., 1998). En particular, se ha reportado la presencia de ambas especies dentro del fitoplancton moderno del Lago Klamath Superior (Oregon, EEUU), un lago localizado en un clima frío (temperatura media anual $9^{\circ} \mathrm{C}$ ), de aguas dulces, circumneutrales a alcalinas, no muy profundas $(\leq 15 \mathrm{~m})$ e hipertróficas (Colman et al., 2004). En este lago estas especies presentan florecimientos durante los períodos de mezcla de la columna de agua, que ocurren en otoño y primavera (Bradbury et al., 2004). En México sólo se ha reportado a $S$. niagarae como una especie abundante en Jilotepec (Oliva-Martínez et al., 2005; Valadez et al., 2005), un sitio de agua dulce, circumnetural, localizado a una altitud de $2560 \mathrm{~m}$ s.n.m., en un clima semi-húmedo (precipitación media de $850 \mathrm{~mm} / \mathrm{año}$ ) y templado (temperatura media anual de $14^{\circ} \mathrm{C}$ ). Ambas especies son frecuentes en el registro fósil de EEUU y Canadá. Por ejemplo, $S$. niagarae es abundante en el registro del EI 6 y del EI 2 del Lago Owens, California (Bradbury, 1997), y durante los climas fríos del EI 2 su registro se extiende hacia el norte y centro de México. Ambas especies son abundantes en sedimentos que datan del último máximo glacial (EI 2) en el Lago de Pátzcuaro, Michoacán (Bradbury, 2000) y S. niagarae está presente durante el

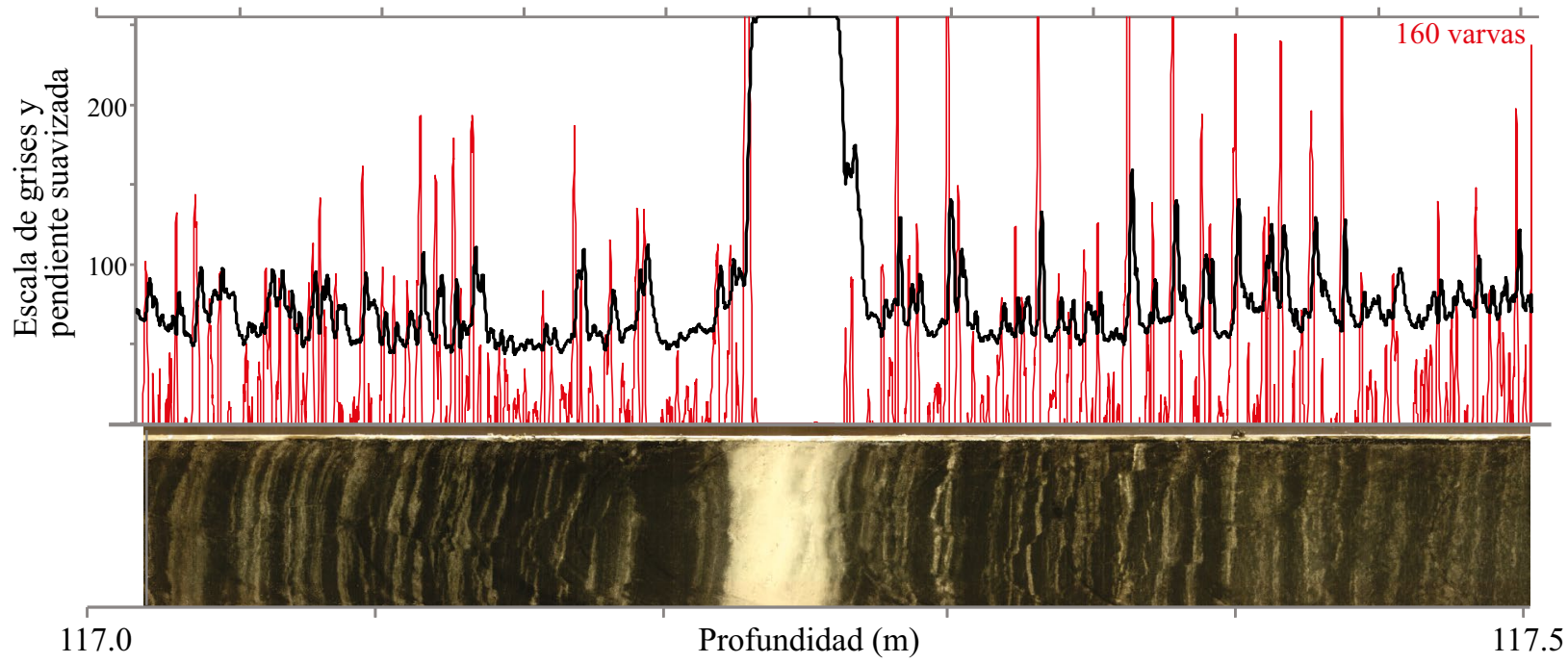

Figura 5. Ejemplo del método para el conteo de varvas, se muestra la escala de grises (negro) y los valores positivos de la pendiente suavizada (rojo), cuyas inflexiones equivalen a las láminas oscuras (160 en total) a lo largo de esta sección de sedimentos laminados del Lago Chalco, cuenca de México. 
Tabla 1. Conteos de láminas oscuras y estimación de tasas de sedimentación para ocho secciones de la secuencia laminada de Chalco, cuenca de México. Las tasas de sedimentación se estimaron considerando que cada par de laminaciones claro-oscuro representa un año de sedimentación y eliminando el espesor de las capas de lodo amarillo pálido en los dos casos (*) en los que este tipo de material estaba intercalado en la secuencia analizada.

\begin{tabular}{|c|c|c|c|}
\hline $\begin{array}{l}\text { Profundidad } \\
\text { (m) }\end{array}$ & $\begin{array}{c}\text { No. de láminas } \\
\text { oscuras }\end{array}$ & $\begin{array}{l}\text { Longitud } \\
\quad(\mathrm{cm})\end{array}$ & $\begin{array}{r}\text { Tasa sedim. } \\
\text { (mm/año) }\end{array}$ \\
\hline $110.14-109.45$ & 242 & 69 & 2.9 \\
\hline $111.27-110.58$ & 225 & 69 & 3.1 \\
\hline $112.43-111.58$ & 284 & 85 & 3.0 \\
\hline $113.55-112.70$ & 258 & 85 & 3.3 \\
\hline $114.58-113.79^{*}$ & 257 & 72 & 2.8 \\
\hline $116.87-116.47$ & 154 & 40 & 2.6 \\
\hline $117.55-117.06^{*}$ & 160 & 46 & 2.9 \\
\hline \multirow[t]{3}{*}{$118.60-118.13$} & 208 & 45 & 2.2 \\
\hline & & Rango & $2.2-3.3$ \\
\hline & & Promedio & 2.9 \\
\hline
\end{tabular}

EI 2 en sedimentos del Lago Babícora, en Chihuahua (Metcalfe et al., 2002) y en sedimentos del Lago de Tecocomulco (Caballero et al., 1999) y del propio Lago de Chalco (Caballero y Ortega-Guerrero, 1998), en la cuenca de México. Sin embargo, la abundancia de esta especie en México decreció dramáticamente con el establecimiento de climas más cálidos durante el Holoceno (EI 1) (Valadez et al., 2005). La distribución actual y pasada de estas especies indica que durante la deposición de la secuencia laminada, el Lago de Chalco era un lago significativamente más profundo que el actual, de aguas dulces, eutróficas a hipertróficas. Estas características son consistentes con la preservación de las laminaciones, al promover condiciones anóxicas y limitar la bioturbación y la re-suspensión de los sedimentos (Zolitschka et al., 2015). En particular, la ecología de estas especies sugiere la presencia de aguas templadas a frías, lo que sustenta la interpretación de que la secuencia laminada de Chalco se depositó durante un intervalo de clima frío como correspondería con el EI 6. Al comparar los sedimentos acumulados en Chalco durante el EI 2 (último máximo glacial) y el EI 6 (penúltimo máximo glacial) es interesante destacar que durante el EI 2 no se depositaron sedimentos laminados similares a los del EI 6 y que la abundancia de $S$. niagarae es baja, comparada con la gran abundancia de esta especie durante el EI 6 (Caballero y Ortega-Guerrero, 1998; Ortega-Guerrero et al., 2017), lo cual sugiere que el EI 6 debió de haber sido más frío y húmedo que el EI 2, como también lo indican los registros glaciares del Iztaccíhuatl (VázquezSelem, 2011; Vázquez-Selem y Heine, 2011).

De manera detallada, en cuanto al tipo de laminaciones presentes en la secuencia laminada de Chalco, se puede inferir lo siguiente: el alto contenido de Ti en las láminas oscuras, en comparación con las láminas claras, es congruente con la dominancia de minerales clásticos. Siendo el Ti un indicador de elevada escorrentía y transporte de terrígenos de la cuenca hacia el lago (Kylander et al., 2011; Corella et al., 2012), se infiere que las láminas oscuras se depositaron en periodos de elevada precipitación y escorrentía superficial. Por otro lado, los altos valores de $\mathrm{Si}$ /Ti en las láminas claras y la gran abundancia de valvas de diatomeas permiten identificarlas como una facies biogénica de lodo (ooze) de diatomeas, si bien también se detecta la presencia de minerales autigénicos, lo que indica que el depósito de esta facies estuvo asociado con florecimientos estacionales de diatomeas durante los cuales la alta tasa de fotosíntesis favoreció la precipitación de estos minerales (Sáez et al., 2007). Se propone, por lo tanto, que las laminaciones claro-oscuro en Chalco representan una secuencia anualmente laminada, con una alternancia de láminas clásticas-biogénicas. Considerando el contexto climatológico moderno de México, proponemos un modelo en el que el depósito de la lámina clástica (oscura) se asocia con una estación de lluvias intensas de verano, mientras que el de la lámina biogénica (clara) corresponde con un florecimiento de diatomeas favorecido por la mezcla invernal de la columna de agua (Figura 6a y 6b). Los florecimientos de diatomeas asociados con una mezcla de la columna de agua que favorece la recirculación de nutrientes son un fenómeno relativamente común, por ejemplo, este mecanismo se ha asociado con florecimientos invernales de Cyclostephanos andinus en el lago Chungará, Chile (Dorador et al., 2003; Bao et al., 2015). En este lago, capas de lodos (ooze) de diatomeas en la secuencia sedimentaria han sido interpretadas como eventos durante los cuales la columna de agua tuvo una mezcla más eficiente (Sáez et al., 2007). En los lagos tropicales monomícticos como los que existen en México, también se han documentado los florecimientos invernales de diatomeas asociados con la mezcla de la columna de agua, como por ejemplo en Santa María del Oro, Nayarit (Caballero et al., 2013) o Alchichica, Puebla (Oliva et al., 2001). La mezcla de la columna de agua también puede ser favorecida por vientos más intensos, los cuales en la cuenca de México frecuentemente coinciden con el invierno (Jáuregui-Ostos y Luyando, 1992). Este tipo de laminación (clástica-biogénica) es más común en ambientes relativamente húmedos, mientras que las de tipo clástica-química corresponden con ambientes más secos (Zolitschka et al., 2015), situación que es consistente con el contexto paleoclimático del EI 6, que representa una etapa durante la cual dominaba un clima frío-húmedo, asociado con una baja evaporación y un lago más
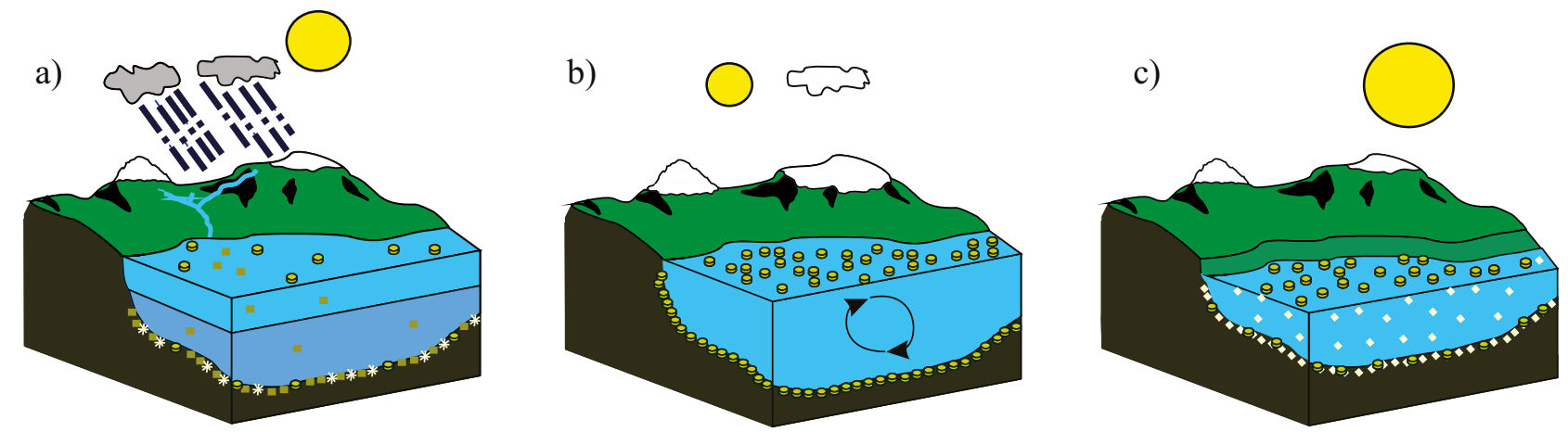

Figura 6. Diagrama del modelo de depósito de las laminaciones claras, oscuras y las capas de lodo micrítico propuesto para el Lago de Chalco, cuenca de México. a) Depósito de la lámina oscura durante la estación de lluvias de verano (lago estratificado) con abundante aporte de terrígenos. b) Depósito de la lámina clara en invierno, durante la mezcla de la columna de agua, que permite la circulación de nutrientes y el florecimiento de diatomeas. c) Depósito de la lámina clara durante primaveras/veranos extremadamente cálidos, cuando una reducción del nivel del lago favorece la circulación de nutrientes y una alta productividad biológica. 
profundo. El ejemplo más cercano de un lago moderno que deposita varvas bajo este modelo sedimentario es el Lago Malawi, un lago tropical profundo $(293 \mathrm{~m})$ en un clima cálido-húmedo $\left(24^{\circ} \mathrm{C}\right.$ media anual, $1630 \mathrm{~mm} / \mathrm{año}$ ), donde una lámina clástica oscura se deposita en temporada de lluvias y una lámina biogénica clara, conformada por valvas de diatomeas de los géneros Aulacoseira y Stephanodiscus, se deposita durante la mezcla invernal (Pilskaln y Johnson, 1991).

Por otro lado, las capas de lodo amarillo pálido se caracterizan por la abundancia de minerales autigénicos como la calcita, lo que es congruente con los altos valores de $\mathrm{Ca} / \mathrm{Ti}$ que presentan, y que permiten definirlas como una facies predominantemente química, de lodo micrítico. Estas capas, sin embargo, tienen abundantes componentes biogénicos, como diatomeas, espículas de esponjas y ostrácodos, lo que sugiere que su depósito estuvo asociado con momentos de intensa evaporación, pero también de una alta productividad en el lago. La presencia de espículas de esponja y de valvas de ostrácodos sugiere que el lago fue, en general, más somero durante el depósito de estas capas que durante el depósito de los sedimentos laminados, un factor que puede favorecer la elevada productividad biológica al reducir el tirante de la columna de agua y facilitar la recirculación de nutrientes (Bao et al., 2015). Se propone que el depósito de estas capas de lodo micrítico, que interrumpen la secuencia laminada, debe estar asociado con eventos cálido-secos durante los cuales hubo una intensa evaporación (ej. veranos muy cálidos), pero también una alta productividad en el lago, de manera que ambos factores favorecieron la precipitación de carbonatos autigénicos. Los eventos que originaron las capas de lodo micrítico parecen ser frecuentes y posiblemente tengan algún patrón de recurrencia que sería necesario estudiar más a fondo. Estos eventos podrían ser de origen climático, pero también podrían estar relacionados con la entrada de aguas subterráneas ricas en Ca por una intensificación de la actividad volcánica e hidrotermalismo en la zona (Sáez et al., 2007). Tomando en cuenta la tasa de sedimentación media de $2.9 \mathrm{~mm} / \mathrm{año}$, estimada por el conteo de láminas, y un espaciamiento estratigráfico entre estas capas de 0.4 a $0.8 \mathrm{~m}$, la recurrencia de estos eventos es de entre 140 a 280 años, lo que sugiere que pudieron estar asociados con los ciclos de 200 años en la actividad solar (ciclo De Vries). La presencia de sedimentos masivos que alternan con los sedimentos laminados y la abundante presencia de valvas de ostrácodos y de una asociación de diatomeas dominada por especies halófilas como Anomoeoneis costata y Campylodiscus clypeus en cinco de las capas de lodo micrítico (Figura 2), sugieren que en Chalco existieron momentos más o menos prolongados durante los cuales el ambiente lacustre era más somero y de mayor salinidad, en alternancia con los ambientes más profundos y de aguas dulces que dominaban durante el depósito de los sedimentos laminados. Estas fluctuaciones ambientales fueron posiblemente de escala milenaria y, junto con el depósito de las capas de lodo micrítico en escalas de $\sim 200$ años, sugieren la existencia de una alta variabilidad ambiental hacia finales del EI 6 y durante la Terminación II (T-II).

El grosor de las laminaciones y las tasas de sedimentación, estimadas mediante sus conteos considerando un modelo de sedimentación anualmente laminado para Chalco ( 2.2 a $3.3 \mathrm{~mm} / \mathrm{año})$, están dentro de los rangos reportados para los lagos anualmente laminados en México y entran dentro de los rangos reportados para la parte superior de la propia secuencia de Chalco (Tabla 2), por lo que, basados en este criterio, el modelo de sedimentación anualmente laminado para Chalco es factible. Sin embargo, las dificultades encontradas en el conteo de las laminaciones hacen que éstos sean solamente estimados máximos y, muy posiblemente, las tasas reales de sedimentación sean menores. La tasa de sedimentación promedio de $2.9 \mathrm{~mm} /$ año permite estimar que los $\sim 15 \mathrm{~m}$ de secuencia laminada de Chalco (excluyendo tefras y capas de lodo micrítico) se depositaron a lo largo de $\sim 5000$ años y no de los 20000 años que se estimó extrapolando la tasa de $0.8 \mathrm{~mm}$ /año (Ortega et al., 2017), lo que modificaría ligeramente la edad de la base de la secuencia de $122.5 \mathrm{~m}$ de Chalco, de alrededor de 150000 años a unos 140000 años. Sin embargo, esto no modifica el contexto general en el que esta inusual secuencia laminada en Chalco se depositó hacia el final del EI 6, muy cerca de la Terminación II.

\section{AGRADECIMIENTOS}

Este trabajo fue financiado por la Universidad Nacional Autónoma de México (UNAM) a través del proyecto DGAPA-PAPIIT-IV-100215 "Cambio Climático y Medio Ambiente en la historia del lago de Chalco". Agradecemos el apoyo de Anders Noren, Amy Mybro y Kristina Brady,

Tabla 2. Tipos de laminaciones y tasas de sedimentación reportadas para diferentes lagos con sedimentos anualmente laminados de México y del Lago Malawi, en África Oriental.

\begin{tabular}{|c|c|c|c|}
\hline $\begin{array}{l}\text { Lago } \\
\text { (prof. max.) }\end{array}$ & Tipo de varvas & $\begin{array}{l}\text { Tasas de sedimentación (mm/año) } \\
\text { mín.-(prom.)-máx. }\end{array}$ & Referencia \\
\hline $\begin{array}{l}\text { Chalco, Ciudad de } \\
\text { México }\end{array}$ & $\begin{array}{l}\text { Sedimentos masivos por } \\
\text { arriba de los } 63.5 \mathrm{~m} \text {. }\end{array}$ & $0.2-(0.8)-12.5$ & Ortega-Guerrero et al., 2017 \\
\hline $\begin{array}{l}\text { Chalco, Ciudad de } \\
\text { México }\end{array}$ & $\begin{array}{l}\text { Sedimentos laminados } \\
\text { Clástico-biogénico }\end{array}$ & $2.2-(2.9)-3.3$ & Este trabajo \\
\hline Aljojuca, Pue. $(30 \mathrm{~m})$ & $\begin{array}{l}\text { Sedimentos laminados } \\
\text { Clástico-químico }\end{array}$ & $1.2-(3.5)-6.2$ & Bhattacharya y Byrne, 2016 \\
\hline Minucúa, Oax. (0.45 cm) & $\begin{array}{l}\text { Sedimentos laminados } \\
\text { Clástico-químico }\end{array}$ & $(1.2)$ & Goman et al., 2017 \\
\hline $\begin{array}{l}\text { Alberca Gto. } \\
(50 \mathrm{~m})\end{array}$ & $\begin{array}{l}\text { Sedimentos laminados } \\
\text { Clástico-químico }\end{array}$ & $0.05-(2.23)-9.3$ & Kienel et al., 2009 \\
\hline $\begin{array}{l}\text { Rincón, Gto. } \\
(50 \mathrm{~m})\end{array}$ & $\begin{array}{l}\text { Sedimentos laminados } \\
\text { Clástico-químico }\end{array}$ & $0.55-(1.43)-4.40$ & Kienel et al., 2009 \\
\hline Juanacatlán, Jal. (25 m) & $\begin{array}{l}\text { Sedimentos laminados } \\
\text { Clástico-biogénico }\end{array}$ & $(1.75)$ & Davies et al., 2005 \\
\hline $\begin{array}{l}\text { Malawi, África Oriental } \\
(>200 \mathrm{~m})\end{array}$ & $\begin{array}{l}\text { Sedimentos laminados } \\
\text { Clástico-biogénico }\end{array}$ & $0.5-(1.0)-1.5$ & Pilsklan y Johnson, 1991 \\
\hline
\end{tabular}


así como de todo el personal del National Lacustrine Core Facility (LacCore) de la Universidad de Minnesota en Minneapolis, agradecemos también el apoyo técnico de Susana Sosa, Cecilia Caballero, Ana María Soler y Teodoro Hernández. Diana Avendaño Villeda agradece el apoyo económico otorgado por el proyecto DGAPA-PAPIIT-IV-100215 para realizar este estudio como parte de su tesis de licenciatura. También agradecemos los valiosos comentarios al artículo realizados por Dr. Mark Brenner, Dr. Blas Valero y un tercer árbitro anónimo.

\section{MATERIAL SUPLEMENTARIO}

Las Tablas S1, S2 y S3, pueden ser descargadas del portal web www.rmcg.unam.mx, en la tabla de contenido de este volumen.

\section{REFERENCIAS:}

Anderson, R.Y., Dean, W.E., 1988, Lacustrine varve formation through time: Palaeogeography Palaeoclimatology Palaeoecology, 62 (1-4), 215-235.

Arce, J., Layer, P., Lassiter, J., Benowitz, J., Macías, J., Ramírez-Espinosa, J., 2013, ${ }^{40} \mathrm{Ar} /{ }^{39} \mathrm{Ar}$ dating, geochemistry, and isotopic analyses of the Quaternary Chichinautzin volcanic field, south of Mexico City: Implications for timing, eruption rate, and distribution of volcanism: Bulletin of Volcanology, 75 (12), 774.

Avendaño-Villeda, D., 2017, Reconstrucción paleolimnológica en el registro del lago de Chalco durante la transición del estadio isotópico marino 6 a 5 (MIS 6 a MIS 5): México, Universidad Nacional Autónoma de México, Facultad de Ciencias, Tesis de Licenciatura, $97 \mathrm{pp}$.

Ayalon, A., Bar-Matthews, M., Kaufman, A., 2002, Climatic conditions during marine oxygen isotope stage 6 in the eastern mediterranean region from the isotopic composition of speleothems of Soreq Cave, Israel: Geology, 30(4), 303-306.

Bao, R., Hernández, A., Sáez, A., Giralt, S., Prego, R., Pueyo, J., Moreno, A., Valero-Garcés, B., 2015, Climatic and lacustrine morphometric controls of diatom paleoproductivity in a tropical Andean lake: Quaternary Science Reviews, 129, 96-110.

Bhattacharya, T., Byrne, R., 2016, Late Holocene anthropogenic and climatic influences on the regional vegetation of Mexico's Cuenca Oriental: Global and Planetary Change, 138, 56-59.

Boës, X., Fagel, N., 2008, Relationships between southern chilean varved lake sediments, precipitation and ENSO for the last 600 years: Journal of Paleolimnology, 39(2), 237-252.

Bradbury, J.P., 1997, A diatom-based paleohydrologic record of climate change for the past 800 ky from Owens lake, California: Geological Society of America Special Paper, 317, 99-112.

Bradbury, J.P., 2000, Limnologic history of lago de Pátzcuaro, Michoacán, Mexico for the past 48,000 years: Impacts of climate and man: Palaeogeography Palaeoclimatology Palaeoecology, 163(1-2), 69-95.

Bradbury, J.P., Colman, S.M., Dean, W.E., 2004, Limnological and climatic environments at Upper Klamath lake, Oregon during the past 45000 years: Journal of Paleolimnology, 31 (2), 167-188.

Brauer, A., 2004, Annually laminated lake sediments and their palaeoclimatic relevance. The climate in historical times: Springer, 109-127.

Brèbisson, A., 1838, Considerations sur les diatomées et essai d'une classification des genres et des espèces appartenant à cette famille. Brée l'Ainé ImprimeurLibraire, Falaise $22 \mathrm{pp}$.

Brook, G.A., Ellwood, B.B., Railsback, L.B., Cowart, J.B., 2006, A 164 ka record of environmental change in the American Southwest from a Carlsbad cavern speleothem: Palaeogeography, Palaeoclimatology, Palaeoecology, 237(2-4), 483-507.

Brugam, R.B., 1983, The relationship between fossil diatom assemblages and limnological conditions: Hydrobiologia, 98(3), 223-235.

Caballero, M., Ortega-Guerrero, B., 1998, Lake levels since about 40,000 years ago at lake Chalco, near Mexico City: Quaternary Research, 50 (1), 69-95.

Caballero, M., Lozano, S., Ortega, B., Urrutia, J., Macias, J.L., 1999, Environmental characteristics of lake Tecocomulco, northern Basin of Mexico, for the last 50,000 years: Journal of Paleolimnology, 22(4), 399-411.

Caballero, M., Rodriguez, A., Vilaclara, G., Ortega, B., Roy, P., Lozano García S., 2013, Hydrochemistry, ostracods and diatoms in a deep, tropical, crater lake in western Mexico: Journal of Limnology, 72(3), 512-523.

Cohen, A.S., 2003, Paleolimnology: The history and evolution of lake systems: New York, Oxford University Press. 500 pp.

Colman, S.M., Bradbury, J.P., McGeehin, J.P., Holmes, C.W., Edginton, D., Sarna-Wojcicki, A.M., 2004, Chronology of sediment deposition in Upper Klamath lake, Oregon: Journal of Paleolimnology, 31(2), 139-149.

Corella, J.P., Brauer, A., Mangili, C., Rull, V., Vegas-Vilarrúbia, T., Morellón, M., Valero-Garcés, B.L., 2012, The 1.5-ka varved record of lake Montcortés (southern Pyrenees, NE Spain): Quaternary Research, 78(2), 323-332.

Davies S., Metcalfe S., Bernal-Brooks F., Chacón-Torres A., Farmer J., MacKenzie A., Newton A., 2005, Lake sediments record sensitivity of two hydrologically closed upland lakes in Mexico to human impact: Ambio 34, 470-475.

De Geer, G., 1912, A geochoronology of the last 12000 years: Congrès de Geologie International, Comptes Rendues, 11, 241-253.

Desmazières, J.B.H.J., 1830, Plantes Cryptogames du nord de la France, 451-500.

Díaz-Rodríguez, J.A., 2006, Los suelos lacustres de la Ciudad de México: Revista Internacional de Desastres Naturales, Accidentes e Infraestructura, 6(2), 111-129.

Dorador, C., Pardo, R., Vila, I., 2003, Variaciones temporales de parámetros físicos, químicos y biológicos de un lago de altura: El caso del lago Chungará: Revista Chilena de Historia Natural, 76(1), 15-22.

Fritz, S.C., Juggins, S., Battarbee, R.W., 1993, Diatom assemblages and ionic characterization of lakes of the Northern Great Plains, North America: A tool for reconstructing past salinity and climate fluctuations: Canadian Journal of Fisheries and Aquatic Sciences, 50(9), 1844-1856.

Goman, M., Joyce, A., Lund, S., Pearson, C., Guerra, W., Dale, D., Hammond, D.E., Celestian, A.J., 2017, Preliminary results from laguna Minucúa a potentially annually resolved record of climate and environmental change for the past $\sim 5000$ years in the Mixteca Alta of Oaxaca, Mexico: Quaternary International. doi: 10.1016/j.quaint.2017.01.027.

Håkansson, H., 1986, A taxonomic reapppraisal of some Stephanodiscus species (Bacillariophyta): British Phycological Journal, 21(1), 25-37.

Herrera-Hernández, D., 2011, Estratigrafía y análisis de facies de los sedimentos lacustres del Cuaternario tardío en la cuenca de Chalco, México, Universidad Nacional Autónoma de México, Posgrado en Ciencias de la Tierra, Tesis de maestría, $122 \mathrm{pp}$.

Hustedt, F., 1959, Die Kieselalgen Deutschlands, Österreichs und der Schweiz unter Berücksichtigung der übrigen Länder Europas sowie der angrenzenden Meeresgebiete. Bd. VII, Teil 2, Liefrung 6, figs 1106-1179: Leipzig, Akademische Verlagsgesellschaft m.b.h., 737-839.

Jáuregui-Ostos, E., Luyando, E., 1992, Patrones de flujo de aire superficial y su relación con el transporte de contaminantes en el valle de México: Investigaciones Geográficas, (24), 51-78.

Julius, M.L., Stoermer, E.F., Taylor, C.M., Schelske, C.L., 1998, Local extirpation of Stephanodiscus niagarae (bacillariophyceae) in the recent limnological record of lake Ontario: Journal of Phycology, 34(5), 766-771.

Kemp, A.E., 1996, Laminated sediments as palaeo-indicators, en Kemps A.E. (ed.), Palaeoclimatology and palaeoceanography from laminated sediments: Geological Society, London, Special Publications,116(1), vii. http://dx.doi.org/10.1144/GSL.SP.1996.116.01.01.

Kienel, U., Bowen, S.W., Byrne, R., Park, J., Bohnel, H., Dulski, P., Luhr, J.F., Siebert, L., Haug, G.H., Negendank, J.F.W., 2009, First lacustrine varve chronologies from Mexico: Impact of droughts, enso and human activity since ad 1840 as recorded in maar sediments from Valle de Santiago: Journal of Paleolimnology, 42(4), 587-609.

Kützing, F.T., 1844, Die Kieselschaligen Bacillarien oder Diatomeen 30 plt. Nordhausen:zu finden bei W. Köhne. 552 pp.

Kylander, M.E., Ampel, L., Wohlfarth, B., Veres, D., 2011, High-resolution x-ray fluorescence core scanning analysis of Les Echets (France) sedimentary sequence: New insights from chemical proxies: Journal of Quaternary Science, 26(1), 109-117.

Lisiecki, L.E., Raymo, M.E., 2005, A Pliocene-Pleistocene stack of 57 globally distributed benthic $\delta^{18} \mathrm{O}$ records: Paleoceanography, 20(1), 1-17.

Litchman, E., Klausmeier, C.A., Yoshiyama, K., 2009, Contrasting size evolution 
in marine and freshwater diatoms: Proceedings of the National Academy of Sciences, 106(8), 2665-2670.

Metcalfe, S., Say, A., Black, S., McCulloch, R., O'Hara, S., 2002, Wet conditions during the last glaciation in the Chihuahuan desert, alta Babicora basin, Mexico: Quaternary Research, 57(1), 91-101.

Metcalfe, S.E., Jones, M.D., Davies, S.J., Noren, A., MacKenzie, A., 2010, Climate variability over the last two millennia in the north american monsoon region, recorded in laminated lake sediments from laguna de Juanacatlán, Mexico: The Holocene, 28(8), 1195-1206.

Mosiño-Alemán, P.A., García, E., 1974, The climate of Mexico, en Bryson R.A.H., Hares F.K. (eds.), Climates of North America: New York, Elsevier, 345-405.

Ojala, A.E.K., Francus, P., Zolitschka, B., Besonen, M., Lamoureux, S.F., 2012, Characteristics of sedimentary varve chronologies - a review: Quaternary Science Reviews, 43, 45-60.

Oliva, G., Lugo, A., Alcocer, J., Peralta, L., Sánchez, M.R., 2001, Phytoplankton dynamics in a deep, tropical, hyposaline lake: Hydrobiologia, 466, 299-306.

Oliva-Martínez, M.G., Ramírez-Martínez, J.G., Garduño-Solórzano, G., Cañetas-Ortega, J., Ortega, M.M., 2005, Caracterización diatomológica en tres cuerpos de agua de los humedales de Jilotepec-Ixtlahuaca, estado de México: Hidrobiologica, 15(1), 01-26.

Ortega-Guerrero, B., Lozano-García, M.S., Herrera-Hernández, D., Caballero, M., Beramendi Orosco, L.E., Bernal, J.P., Torres-Rodríguez, E., AvendañoVilleda, D., 2017, Lithostratigraphy and physical properties of lacustrine sediments of the last ca. $150 \mathrm{kyr}$ from Chalco basin, central México: Journal of South American Earth Sciences, 79, 507-524.

Pantocsek, J., 1902, Kieselalgen oder Bacillarien des Balaton. Resultate der Wissenschaftlichen Erforschung des Balatonsees, herausgegeben von der Balatonsee-Commission der Ung. Geographischen Gesellschaft. Bd 2(2): Wien (Vienna), Commissionsverlag von Ed. Hölzel, 143 pp.

Pilskaln, C.H., Johnson, T.C., 1991, Seasonal signals in lake Malawi sediments: Limnology and Oceanography, 36(3), 544-557.

Reheis, M.C., Adams, K.D., Oviatt, C.G., Bacon, S.N., 2014, Pluvial lakes in the Great Basin of the western United States - a view from the outcrop: Quaternary Science Reviews, 97, 33-57.

Rodríguez-Castillo, R., González-Morán, T., 1989, Comportamiento hidrodinámico del sistema acuífero de la subcuenca de Chalco, México: Geofísica Internacional, 28(2), 207-217.

Roucoux, K., Tzedakis, P., Lawson, I., Margari, V., 2011, Vegetation history of the penultimate glacial period (marine isotope stage 6) at Ioannina, north-west Greece: Journal of Quaternary Science, 26(6), 616-626.
Sáez, A., Valero-Garcés, B.L., Moreno, A., Bao, R., Pueyo, J., González-Sampériz, P., Giralt, S., Taberner, C., Herrera, C., Gibert, R., 2007, Lacustrine sedimentation in active volcanic settings: The late Quaternary depositional evolution of lake Chungará (northern Chile): Sedimentology, 54(5), 1191-1222.

Schneider, C.A., Rasband, W.S., Eliceiri, K.W., 2012, Nih image to Image j: 25 years of imgae analysis: Nature Methods, 9, 671-675.

Schnurrenberger, D., Russell, J., Kelts, K., 2003, Classification of lacustrine sediments based on sedimentary components: Journal of Paleolimnology, 29 (2), 141-154.

Theriot, E., Stoermer, E.F., 1981, Some aspects of morphological variation in Stephanodiscus niagarae (Bacillariophyceae): Journal of Phycology, 17(1), 64-72.

Theriot, E., Stoermer, E.F., 1984, Principal component analysis of Stephanodiscus: Observations on two new species from the Stephanodiscus niagarae complex: Bacillaria, 7, 37-58.

Torres-Rodríguez, E., Lozano-García, S., Roy, P., Ortega, B., Beramendi-Orosco, L., Correa-Metrio, A., Caballero, M., 2015, Last glacial droughts and fire regimes in the central mexican highlands: Journal of Quaternary Science, 30(1), 88-99.

Valadez, F., Oliva, G., Vilaclara, G., Caballero, M., Rodríguez, D.C., 2005, On the presence of Stephanodiscus niagarae Ehrenberg in central Mexico: Journal of Paleolimnology, 34(2), 147-157.

Vázquez-Selem, L., 2011, Las glaciaciones en las montañas del centro de México, en Caballero M., Ortegas B. (eds.), Escenarios de cambio climático: Registros del Cuaternario en América Latina I: México Universidad Nacional Autónoma de México 215-238.

Vázquez-Selem, L., Heine, K., 2011, Late Quaternayr glaciation in Mexico, en Ehlers, J., Ehlers, J., Gibbard, P.L., Hughess, P.D. (eds.), Quaternary glaciations-extent and chronology: A closer look: Amsterdam, Elsevier, 849-861.

Zolitschka, B., Francus, P., Ojala, A.E.K., Schimmelmann, A., 2015, Varves in lake sediments - a review: Quaternary Science Reviews, 117, 1-41.

Manuscrito recibido: enero 9, 2018

Manuscrito corregido recibido: abril 16, 2018

Manuscrito aceptado: abril 16, 2018 